\title{
Can we predict the outcome for people with patellofemoral pain? A systematic review on prognostic factors and treatment effect modifiers
}

\author{
M Matthews, ${ }_{1}^{1}$ M S Rathleff, ${ }^{2,3}$ A Claus, ${ }^{1}$ T McPoil, ${ }^{4}$ R Nee, ${ }_{1}^{5}$ K Crossley, ${ }^{6}$ \\ B Vicenzino $^{1}$
}

- Additional material is published online only. To view please visit the journal online (http://dx.doi.org/10.1136/ bjsports-2016-096545).

${ }^{1}$ Sports Injuries Rehabilitation and Prevention for Health Research Unit, The University of Queensland, School of Health and Rehabilitation Sciences, Brisbane, Queensland, Australia ${ }^{2}$ Research Unit for General Practice in Aalborg and Department of Clinical Medicine, Aalborg, Denmark

${ }^{3} \mathrm{SMI}$, Department of Health Science and Technology, Faculty of Medicine, Aalborg University, Aalborg, Denmark

${ }^{4}$ School of Physical Therapy, Rueckert-Hartman College for Health Professions, Regis University, Denver, Colorado, USA

${ }^{5}$ School of Physical Therapy, Pacific University, Hillsboro, Oregon, USA

${ }^{6}$ La Trobe University, La Trobe Sport and Exercise Medicine Research Centre, Melbourne, Victoria, Australia

Correspondence to Professor B Vicenzino, Sports Injuries Rehabilitation and Prevention for Health Research Unit, The University of Queensland, School of Health and Rehabilitation Sciences, CCRE Spine, Brisbane, QLD 4072, Australia;

b.vicenzino@uq.edu.au

Accepted 18 November 2016 Published Online First 12 December 2016

\section{CrossMark}

To cite: Matthews $M$, Rathleff MS, Claus A, et al. Br J Sports Med 2017:51:1650-1660.

\section{ABSTRACT}

Background Patellofemoral pain (PFP) is a multifactorial and often persistent knee condition. One strategy to enhance patient outcomes is using clinically assessable patient characteristics to predict the outcome and match a specific treatment to an individual.

Aim A systematic review was conducted to determine which baseline patient characteristics were (1) associated with patient outcome (prognosis); or (2) modified patient outcome from a specific treatment (treatment effect modifiers).

Methods 6 electronic databases were searched (July 2016) for studies evaluating the association between those with PFP, their characteristics and outcome. All studies were appraised using the Epidemiological Appraisal Instrument. Studies that aimed to identify treatment effect modifiers underwent a checklist for methodological quality.

Results The 24 included studies evaluated 180 participant characteristics. 12 studies investigated prognosis, and 12 studies investigated potential treatment effect modifiers. Important methodological limitations were identified. Some prognostic studies used a retrospective design. Studies aiming to identify treatment effect modifiers often analysed too many variables for the limiting sample size and typically failed to use a control or comparator treatment group. 16 factors were reported to be associated with a poor outcome, with longer duration of symptoms the most reported ( $>4$ months). Preliminary evidence suggests increased midfoot mobility may predict those who have a successful outcome to foot orthoses.

Conclusions Current evidence can identify those with increased risk of a poor outcome, but methodological limitations make it difficult to predict the outcome after one specific treatment compared with another. Adequately designed randomised trials are needed to identify treatment effect modifiers.

\section{INTRODUCTION}

Patellofemoral pain (PFP) is a prevalent and persistent knee condition ${ }^{1}{ }^{2}$ that affects $\sim 1$ in 20 teenagers and 1 in 10 adult women. ${ }^{3-7}$ Despite receiving evidence-based treatments that are initially effective, more than one-third of patients' still report persistent symptoms 12 months later ${ }^{2}$ with $\sim 25 \%$ reporting symptoms up to 20 years later. ${ }^{8}$ It might be helpful clinically to know whether certain prognostic factors can identify patients with PFP who are at risk for a poor outcome. A review identified a number of prognostic factors for outcomes in those with PFP (eg, age, pain severity, foot posture/motion), ${ }^{9}$ but only presented differences between groups at baseline, which are not helpful to the clinician wanting to determine the prognosis of a specific patient.

The complex and multifactorial nature of PFP leads to a heterogeneous clinical presentation..$^{10} \mathrm{~A}$ recent best practice guide recommended that treatment be tailored to each patient's presentation, but it did not provide direction for the clinician on how to individually tailor treatment. ${ }^{11}$ Prognostic factors are patient characteristics that help to determine a specific outcome within a certain time period. ${ }^{12} 13$ Treatment effect modifiers are patient characteristics that predict a successful outcome from a specific treatment. An evidence-based approach to individually tailor treatment requires the identification of treatment effect modifiers, because although prognostic factors help predict the likelihood of an outcome within a certain time period, they cannot predict the likelihood of an outcome after a specific treatment. ${ }^{14}$

To inform clinical practice and research related to PFP, the purpose of this systematic review was to determine which baseline patient characteristics were: (1) associated with a poor outcome (prognostic factors); or (2) associated with a successful outcome after a specific treatment (treatment effect modifiers).

\section{METHODS}

\section{Search strategy}

The systematic review was conducted following the PRISMA guideline. ${ }^{15}$ Electronic databases (MEDLINE, Scopus, Embase, CINAHL, SPORTDiscus and Web of Science) were searched up to July 2016 for studies investigating conservative (non-surgical) treatments for PFP. Key search terms relating to PFP and other such synonyms used in all databases were adapted from similar search strategies. ${ }^{10} \quad{ }^{16} \quad 17$ Keywords used to narrow the search to the aim of the review were success*, factor*, predict*, charact*, prognos*. Searches were limited to human studies with no language restrictions (see online supplementary appendix 1). The protocol for the systematic review was not registered.

\section{Eligibility}

Studies were included if they had investigated: (1) participants diagnosed with PFP determined by clinicians based on the report of retro or peripatellar pain that was provoked by either a partial squat, stair ascent or descent and pain reported during palpation of periarticular structures, and (2) an association between patient characteristics that were 
measured at the outset of the study and the outcome (status of the condition) at a later time (minimum period of 1 week). Only conservative (non-surgical) approaches were included. Studies were excluded if they included pain from structures other than the patellofemoral joint, and other knee pathologies such as internal derangement, knee ligament insufficiency or patellar tendinopathy. Case reports or reviews of the literature were also excluded.

\section{Review process}

All identified studies were imported into Endnote X6 (Thomson Reuters, Carlsbad, California, USA) and duplicates removed. Two reviewers (MM and MSR) independently assessed study titles and abstracts for eligibility with a third reviewer (BV) available if necessary to resolve discrepancies. Where there was duplication or pooling of data from different trials, only the primary publication (the study of the highest relevancy to the purposes of this review as determined by all three reviewers) was included. Reference lists of all publications considered for inclusion were hand-searched recursively until no additional eligible publications were identified.

\section{Quality assessment}

Two reviewers (MM and MSR) independently assessed papers for quality. Any discrepancies were discussed to reach consensus, and if discrepancies remained, a third reviewer was consulted (BV). Study quality of all included studies was assessed using the Epidemiological Appraisal Instrument $(\mathrm{EAI})^{18}$ in a method used in previous reviews. ${ }^{19}{ }^{20}$ Items were scored as Yes $($ score $=2)$, Partial $($ score $=1)$, No $($ score $=0)$, Unable to determine $($ score $=0$ ) of the applicable items. An average score was then calculated across all applicable items for each study (range 0-2). The EAI is a valid and reliable appraisal instrument for systematic reviews. $^{21}$

Studies that aimed to investigate predictors of outcome after a specific treatment were further evaluated for quality using a checklist for prescriptive, derivation-based clinical prediction rules (QUADCPR). ${ }^{22}$ The QUADCPR was designed and developed using a three-round Delphi process involving physicians, epidemiologists and physical therapists. It includes 23 items across 4 sections-(1) sample and participants, (2) outcome measure, (3) quality of tests and measures and (4) statistical assumptions. Each item is scored yes, no or unclear without generating a quantitative score. Two modifications were made to the QUADCPR for the purposes of this review. First, in accordance with the statement on Transparent Reporting of a multivariable prediction model for Individual Prognosis or Diagnosis (TRIPOD), ${ }^{23}$ an adequately powered study required at least 10 participants in the limiting sample size (group with least frequent outcome) for each variable analysed as a potential predictor (Question 18). Second, in discussion with the corresponding author of the QUADCPR, a fifth section was added to assess whether outcomes are treatment effect modifiers-(5) quality of treatment approach. This section addressed the quality of the treatment approach using published recommendations on the preferred study methods for identifying treatment effect modifiers and subgroup effects. ${ }^{14} 2425$ An additional four questions were inserted into the checklist (Questions 24-27) that addressed treatment explanation and implementation of the target treatment and comparator treatment.

\section{Data extraction and analysis}

Study details were extracted by MM and checked by MSR. Details extracted were: publication details, sample characteristics, participant demographics, study methods, including study design, outcome measures, any intervention(s) and the baseline factors studied. Study results were extracted by following the definitions for a successful outcome or poor outcome applied by each individual study. Outcome measures used per study are detailed in table 1 (col. 4) and table 2 (col. 3). Relationships between baseline predictors and a poor outcome (ie, prognosis) were expressed as $\mathrm{R}^{2}$, whereas baseline predictors and a successful outcome after a specific treatment were quantified by extracting positive likelihood ratios $(\mathrm{LR}+)$ and ORs. Positive likelihood ratios indicate the probability of having a successful outcome if the identified predictor is present. Shifts in probability of a successful outcome are categorised as small and rarely important $(\mathrm{LR}+1-2)$, small but sometimes important $(\mathrm{LR}+2-5)$, moderate shift $(\mathrm{LR}+5-10)$ or large and often conclusive $(\mathrm{LR}+>10) .{ }^{26}$ ORs measure the association between the exposure and an outcome (OR $>1$ higher odds; $\mathrm{OR}<1$ lower odds). For studies that did not report OR, LR+ or post-test probability scores, authors were contacted and those indices were calculated from available data. Meta-analyses were performed where appropriate.

\section{RESULTS}

\section{Search results and critical appraisal of methods}

The search retrieved 11629 citations, of which 7339 unique titles and abstracts were reviewed, with 59 papers identified for full text examination. Twenty-four studies met the eligibility criteria for quality assessment and data extraction (figure 1), which evaluated 180 participant characteristics (see online supplementary appendix 2). The most frequently evaluated characteristics were age and sex $(n=14$ studies $)$, knee pain duration $(n=13), Q$ angle, body mass index, weight and height $(n=8)$, sports participation $(n=6)$ and navicular drop $(n=5$ studies). Twelve studies investigated patient characteristics associated with a poor outcome, and the remaining 12 studies investigated patient characteristics associated with successful outcome after a specific treatment.

\section{Quality assessment}

Overall across all 24 studies, there was good conformity of the study aims, treatments, assessments and main findings on the EAI checklist. Very few studies reported adequate adjustment for covariates in the statistical analyses, blinding of observers or reporting of adverse events. There was also a lack of reporting reliability and validity of the main outcome measures used (see online supplementary appendix 3). Twelve studies investigated prognostic factors for a poor outcome (three randomised controlled trials, eight case series and one had no treatment), only one of the randomised trials ${ }^{27}$ adjusted for treatment and reported it was not a confounder.

In addition to being evaluated for quality on the EAI, studies that investigated patient characteristics associated with successful outcome after a specific treatment were appraised under the QUADCPR checklist $(n=12)$. Overall on the QUADCPR quality checklist, significant methodological limitations were identified with only one study using a control group. Only one study ${ }^{28}$ had adequate statistical power with at least 10 participants in the limiting sample size (group with least frequent outcome) for each potential predictor included in the statistical analysis. Even if a lower threshold of five participants in the limiting sample size for each potential predictor is used, ${ }^{29}$ this was still the only study that had adequate statistical power. An issue with potential reporting bias was identified with only $4^{28} \quad 30-32$ of the 12 studies administered outcome measures in a blinded fashion and 


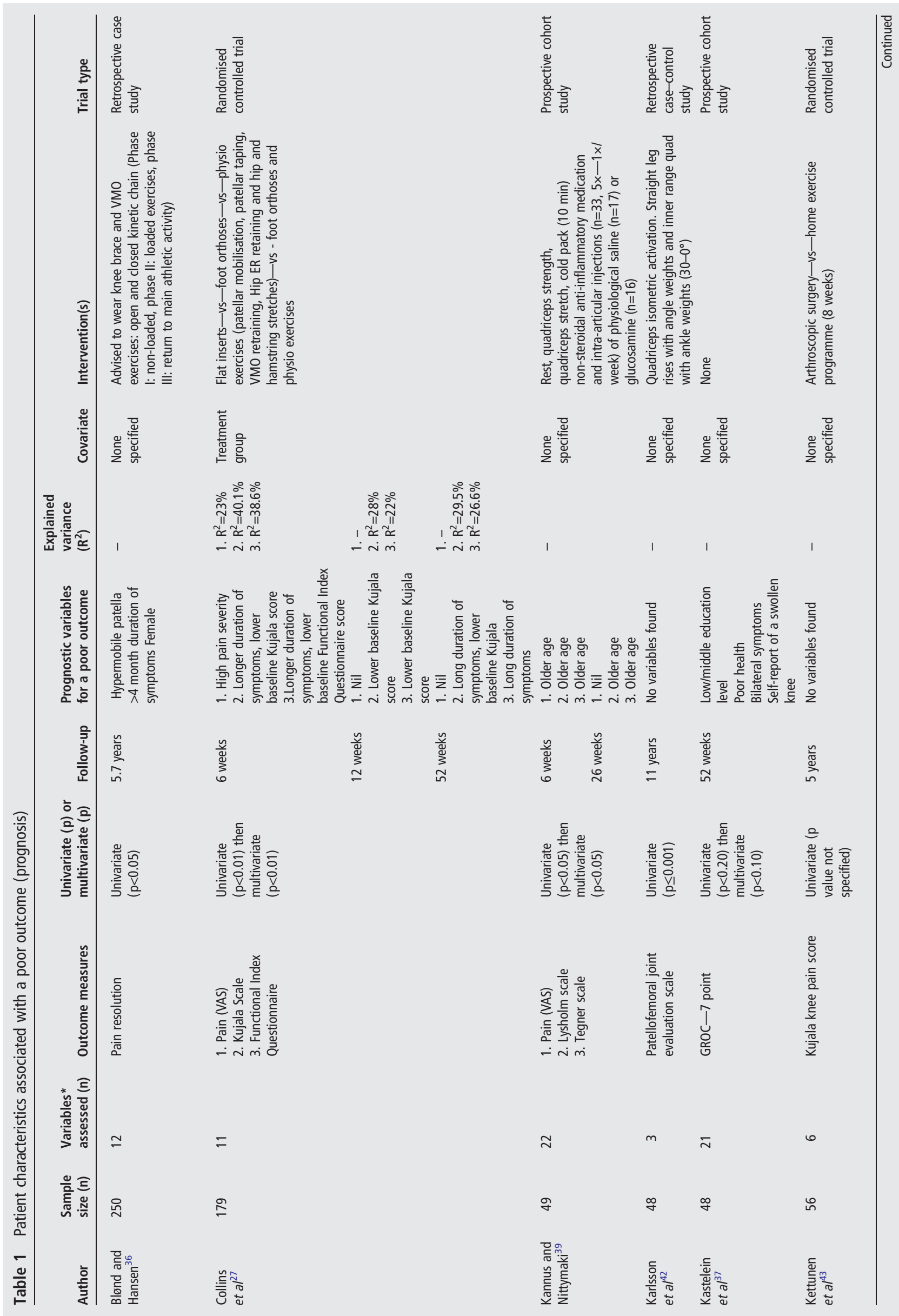




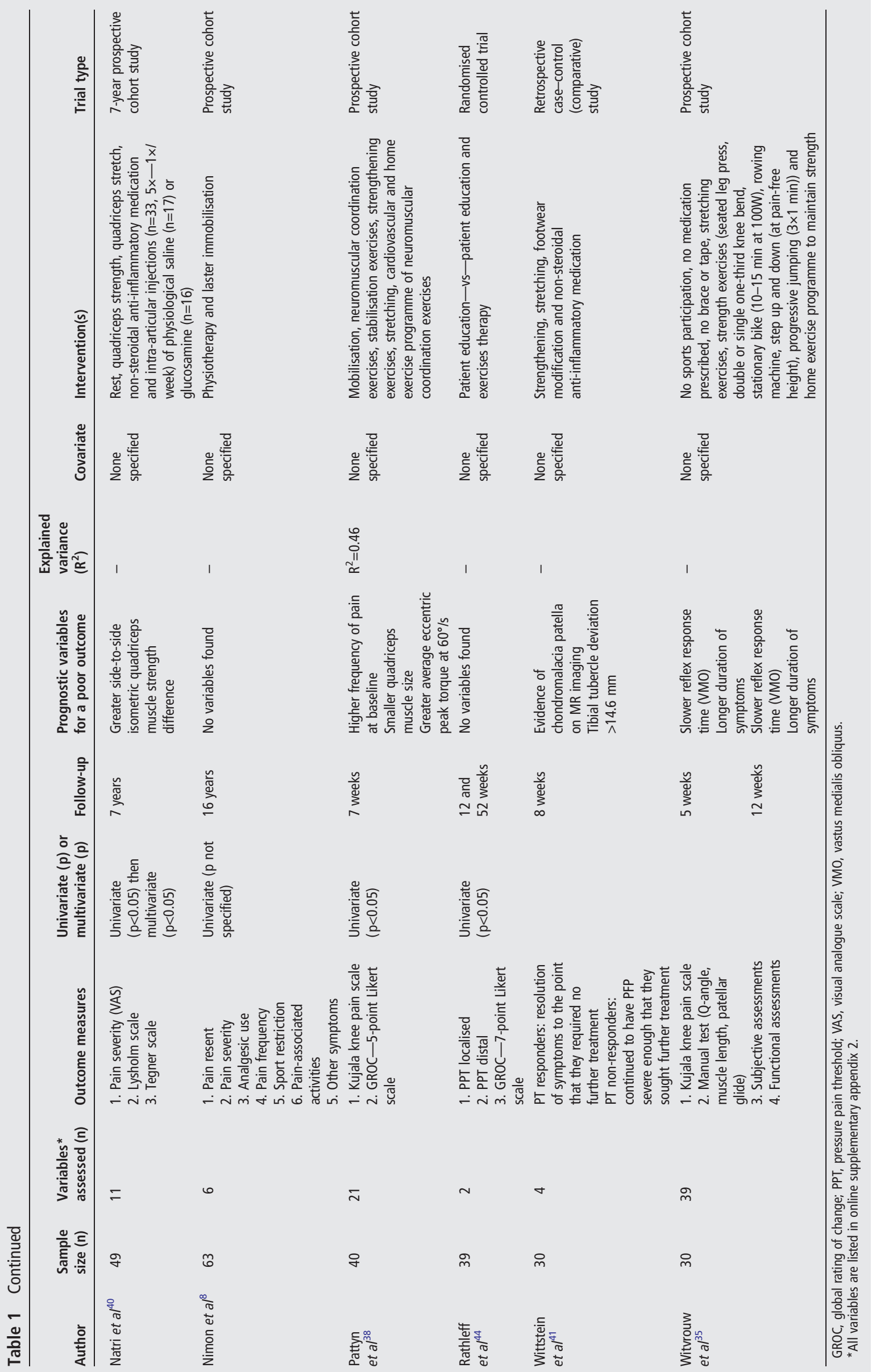




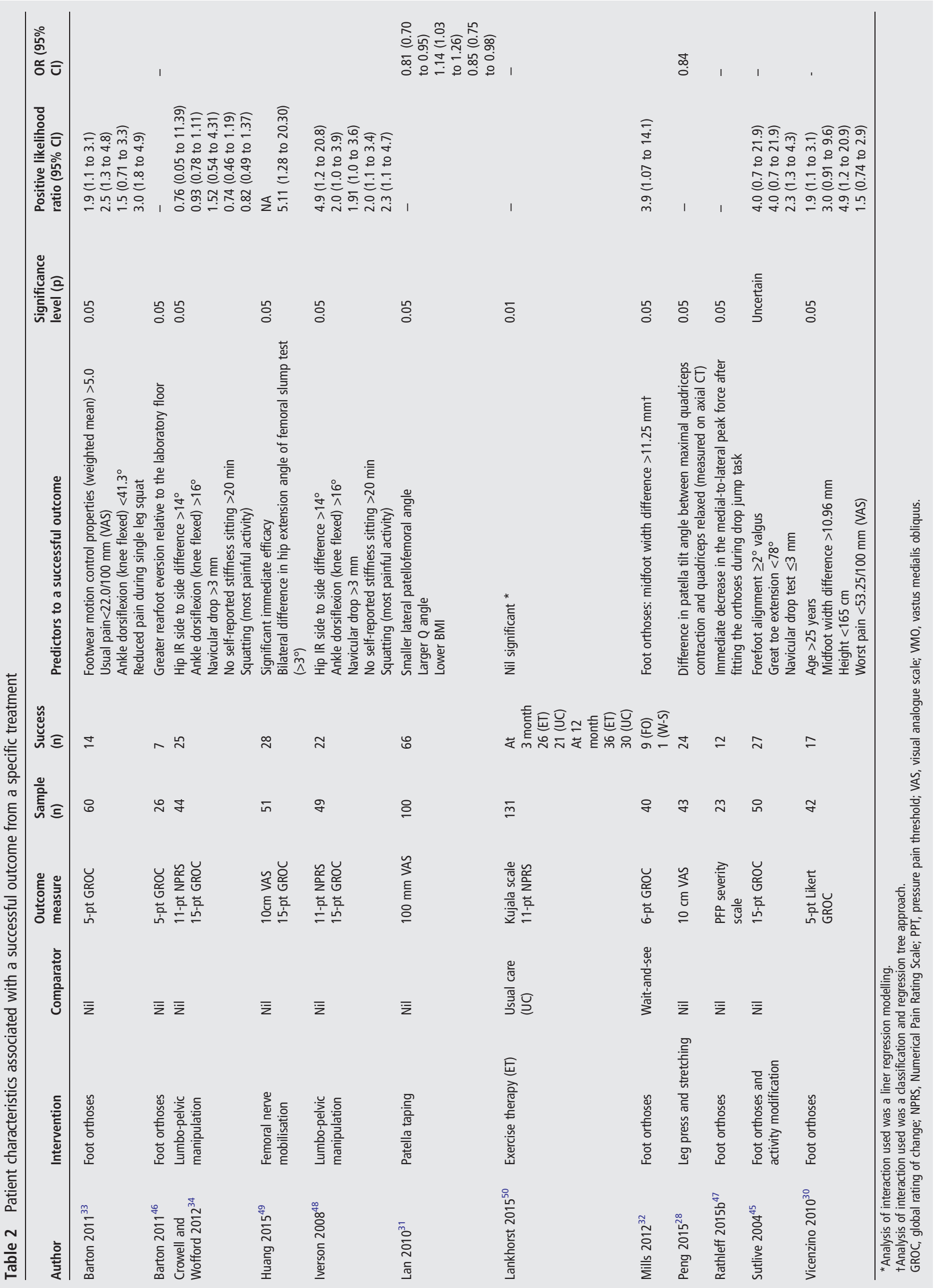




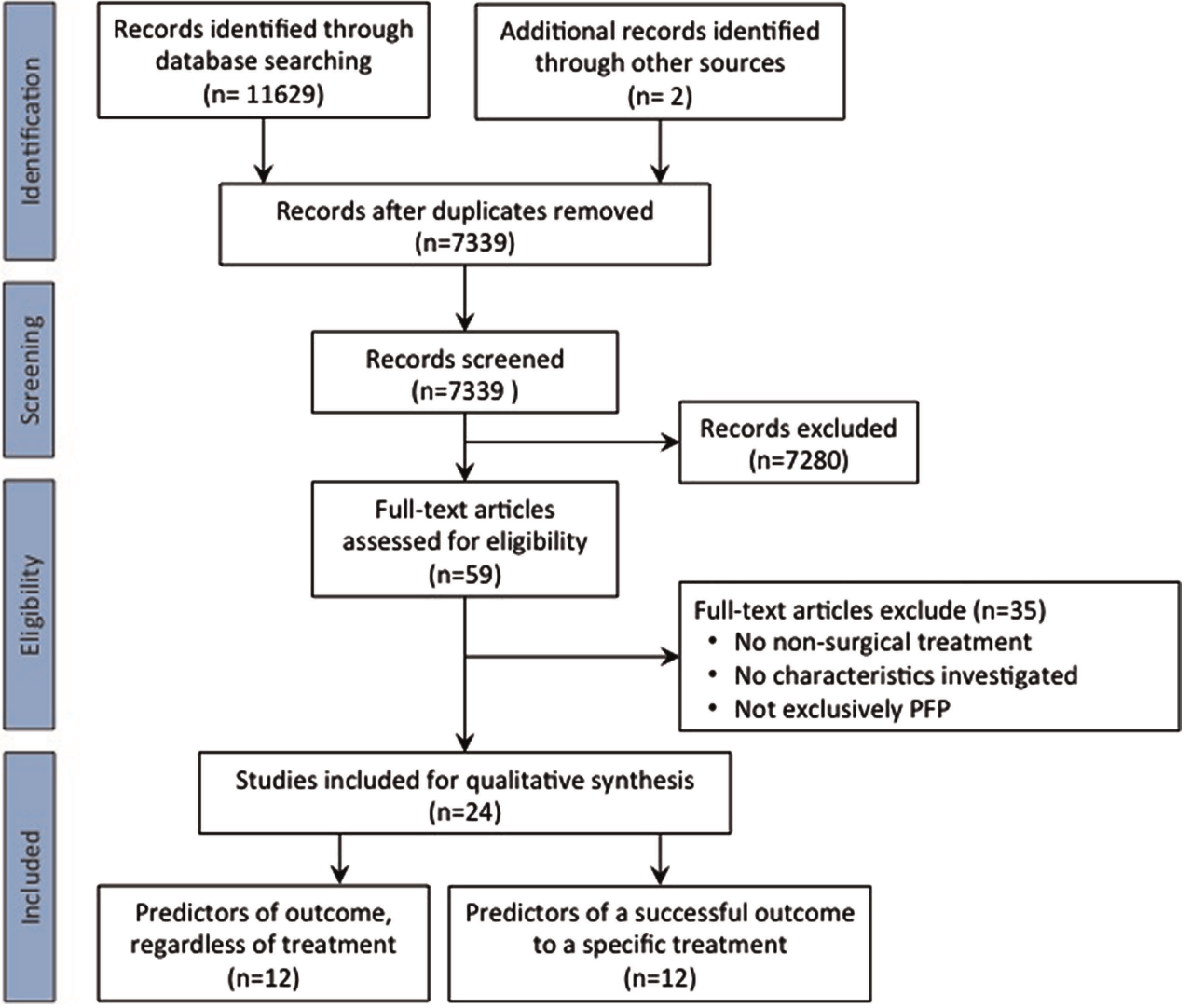

Figure 1 PRISMA flow diagram.

in only 3 studies were the examining ${ }^{28} 3033$ or treating 303234 clinicians blind to the outcome measures (table 3).

\section{Patient characteristics associated with a poor outcome (prognosis)}

Sixteen patient-reported and anatomical characteristics were associated with a poor outcome, with degree of association (eg, $\mathrm{R}^{2}$ ) ranging from $27 \%$ to $46 \%$ (table 1). The patient-reported characteristics were duration of PFP symptoms, ${ }^{27} 3536$ bilateral symptoms, ${ }^{37}$ higher frequency of pain occurrence, ${ }^{38}$ older age, ${ }^{39}$ female gender, ${ }^{36}$ lower baseline Kujala knee pain score and function (Kujala scale and functional index questionnaire in $^{27}$ ), poor health and low/middle education level. ${ }^{37}$

Anatomical characteristics associated with a poor outcome were swelling of the knee (self-reported by the participant in ${ }^{37}$ ), patellar hypermobility, ${ }^{36}$ slower vastus medialis obliquus reflex response, ${ }^{35}$ larger side to side differences in isometric quadriceps muscle strength, ${ }^{40}$ smaller quadriceps cross-sectional area on MRI and lower eccentric knee strength, ${ }^{38}$ evidence of chondromalacia patella on MRI and a tibial tubercle lateral deviation $>14.6 \mathrm{~mm}$ relative to the trochlear groove. ${ }^{41}$

\section{Patient characteristics associated with a successful outcome after a specific treatment}

Six different specific treatments were investigated; foot orthoses, ${ }^{30} 32 \quad 33{ }^{45-47}$ lumbopelvic manipulation, ${ }^{34} 48$ patellar taping, ${ }^{31}$ femoral nerve mobilisation, ${ }^{49}$ leg press exercise and stretching $^{28}$ and exercise therapy (consisting of static and dynamic exercises for the quadriceps muscles, flexibility and balance exercises) ${ }^{50}$ (table 2). Twenty-two patient characteristics were reported to be associated with a successful outcome after a specific treatment. Studies defined a successful outcome using a predetermined amount of improvement in pain scores, questionnaire and/or a rating on a global rating of changes scale to stratify respondents into successful or unsuccessful outcomes.

\section{Foot orthoses}

Fourteen predictors were univariately associated with a successful outcome after foot orthoses treatment across six studies. $^{30} 323345-47$ Four predictors were static measures of the foot; these were $2^{\circ}$ or more of valgus forefoot alignment, $78^{\circ}$ or less great toe extension, $3 \mathrm{~mm}$ or less navicular drop ${ }^{45}$ and two studies reporting a midfoot width difference between weightbearing and non-weight-bearing $>10.96 \mathrm{~mm}^{30}{ }^{32}$ Two studies investigated foot movements during functional tasks. One study used 3D kinematic analysis during gait to show those who had a successful outcome had a mean difference of $2.3^{\circ}$ greater rearfoot eversion relative to the ground than those who reported an unsuccessful outcome. ${ }^{46}$ The other study of a drop jump task reported those who had an immediate decrease in the medial-to-lateral peak foot loading went on to report improvements in pain and function after wearing foot orthoses for 12 weeks. ${ }^{47}$ Two studies found baseline pain scores of usual pain $<22.0 \mathrm{~mm}^{33}$ and worst pain $<53.25 \mathrm{~mm}^{30}$ on a $100 \mathrm{~mm}$ visual analogue scale predicted a successful outcome. One functional performance predictor was reduced pain during a single leg squat while wearing foot orthoses. ${ }^{33}$ Ankle dorsiflexion range $<41.3^{\circ}$, relative to the vertical, (measured as tibial inclination using a digital inclinometer placed anteriorly midtibia) during weight-bearing ankle dorsiflexion with a bent knee also predicted a positive outcome. ${ }^{33}$ The clinical utility of such a specific cut point has not been further studied or the finding replicated. Other demographic predictors reported were height $<165 \mathrm{~cm}$ and age over 25 years. ${ }^{30}$ In addition to the patient 


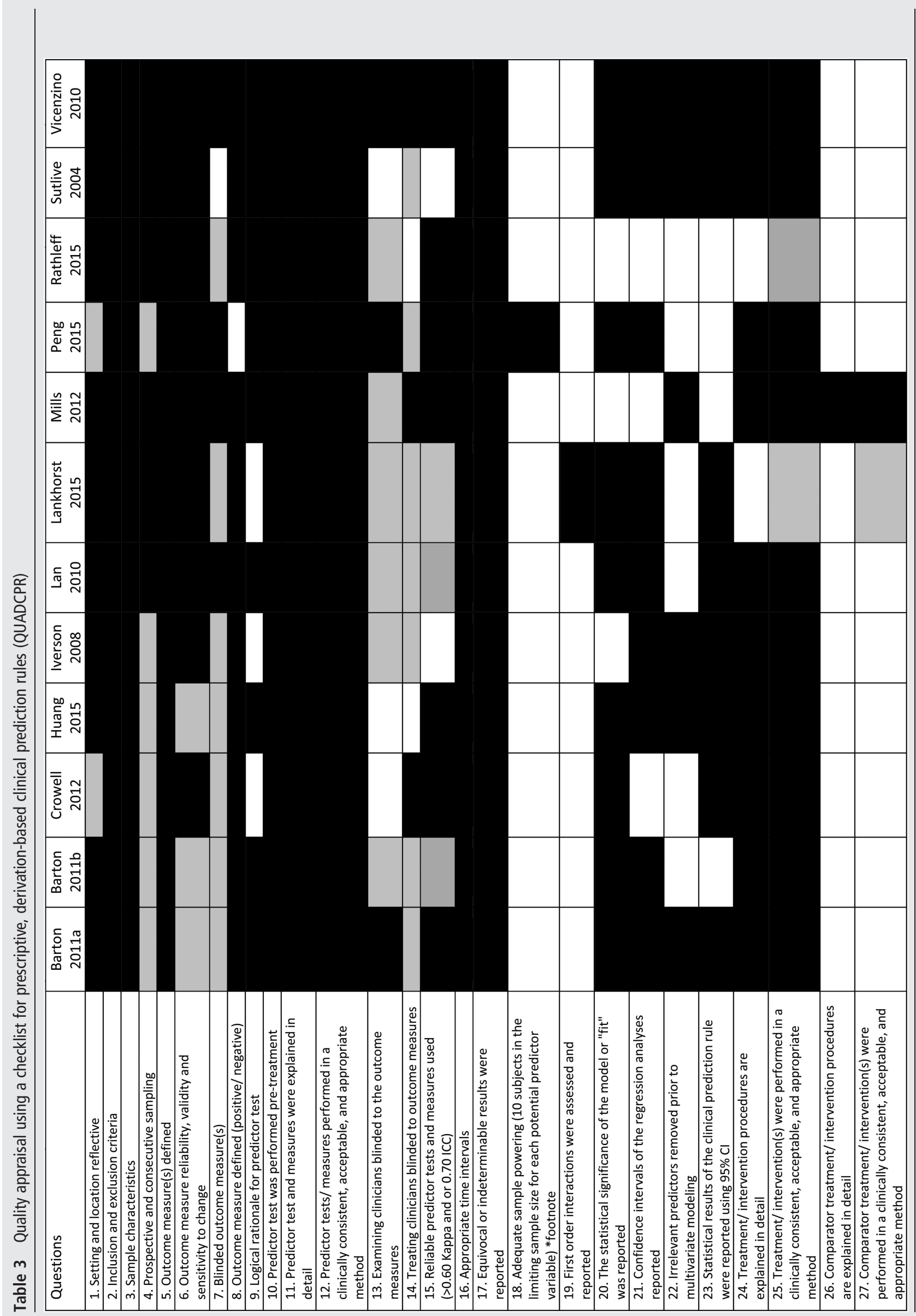

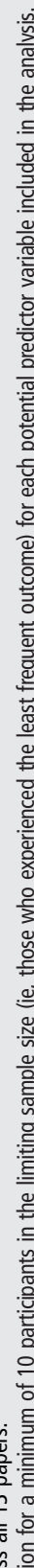

은 흔

के

흥 ठे

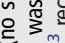

尺 
centric factors, those participants who wore footwear with reduced motion control properties, assessed using a footwear assessment tool, ${ }^{51}$ were more likely to report a successful outcome when wearing an orthosis. ${ }^{33}$

Three predictors (height, forefoot valgus alignment, great toe extension) had a reported LR+ range of $4.0-4.9,3045$ but the 95\% CIs for these predictors were large raising greater uncertainty on the precision of these relationships. Three predictors of success had LR+ range between 2.5 and 3.9 and narrow CIs; with two studies identifying midfoot width difference from weight-bearing to non-weight-bearing foot posture of $>10.96 \mathrm{~mm}^{30}$ and $>11.26 \mathrm{~mm}^{32}$ reduced pain during single leg squat while wearing a foot orthosis and usual pain $<22 /$ $100 \mathrm{~mm}$ visual analogue scale.

Two studies used multivariate analysis to evaluate a combination of predictors for clinical prediction rules for success after treatment by foot orthoses (table 4). Each study reported a different combination of four predictors, with an LR+ of 8.8 $(95 \% \text { CI } 1.2 \text { to } 66.9)^{30}$ and $11.1(95 \% \text { CI } 2.7 \text { to } 46.9)^{33}$ when three or more predictors were present, raising post-test probability of success to $85.4 \%$ and $78 \%$, respectively. These LR+ suggest a moderate to large and often conclusive shifts in probability of a successful outcome after foot orthoses treatment. No participants in either of the two studies presented with all four of the respective predictors (table 4).

\section{Lumbopelvic manipulation}

Five predictors were identified using multivariate analysis and reported to be associated with a successful outcome after lumbopelvic manipulation. ${ }^{48} \mathrm{~A}$ follow-up study ${ }^{34}$ on a different but similar-sized PFP cohort failed to replicate the same five predictors reported by Iverson $e t$ al. $^{48}$

\section{Patellar taping}

One study ${ }^{31}$ reported three predictors of a successful outcome with patellar taping as described by McConnell. ${ }^{52}$ After multivariate analysis, smaller lateral patellofemoral angle (an angle formed by the line between the femoral condyles and another line between the margins of the lateral facet of the patella) measured with radiographs in $30^{\circ}$ of knee flexion, larger $\mathrm{Q}$ angle and a lower body mass index ${ }^{31}$ were reported to be associated with a successful outcome. Larger $\mathrm{Q}$ angle was the only one of the three reported predictors that have an OR $>1$ (OR 1.14 95\% CI 1.03 to 1.26$)$.

\section{Femoral nerve mobilisation}

One study ${ }^{49}$ reported two predictors to be associated with a successful outcome after six sessions of femoral nerve mobilisation. After multivariate analysis, significant immediate improvement after a femoral nerve mobilisation and a bilateral difference of at least $3^{\circ}$ in the hip extension angle of the femoral slump test, which had an LR+ 5.1 (1.3 to 20.3), suggests a moderate shift in probability of successful outcome after femoral nerve mobilisation.

\section{Exercise}

Two studies investigated predictors of successful outcome after exercise. One study compared exercise therapy with usual care and found no significant predictors of a successful outcome to exercise therapy. ${ }^{50}$ One study evaluated a leg press training and lower limb muscle stretching exercise programme. ${ }^{28}$ Patellar tilt angle difference (PTA-d), which is the difference in patellar tilt angle in a quadriceps contracted $(\mathrm{Qc})$ and a quadriceps relaxed $(\mathrm{Qr})$ position measured on axial $\mathrm{CT}$, was associated with a successful outcome after a leg press strengthening and lower limb stretching programme. ${ }^{28}$ Those who had greater PTA-d (ie, greater realignment of the patella with quadriceps contraction) before beginning exercise treatment had greater reductions in pain after treatment. ${ }^{28}$ The optimal cut-off value was $-1.5^{\circ}$ PTA-d (Qc-Qr) for the clinical discrimination of treatment success based on a minimum pain reduction of $1.5 \mathrm{~cm}$ on the VAS (sensitivity $=0.74$, specificity $=0.71, L R+2.5$ ).

\section{DISCUSSION}

Our systematic review on PFP investigated baseline patient characteristics that were associated with a: (1) poor outcome (prognosis), or (2) successful outcome after a specific treatment (treatment effect modifiers) after more than 1 week. The review highlighted a large amount of non-significant association with a total of 180 patient characteristics being investigated by 24 studies. Twelve prognosis studies investigated 104 characteristics and identified 16 prognostic factors associated with a poor outcome. Twelve studies reported that only 22 out of 100 potential treatment effect modifiers were associated with a successful outcome after a specific treatment. However, the review identified significant methodological limitations in all studies appraised with the EAI and modified QUADCPR tools. Of the 12 studies that investigated prognostic factors, only 1 study, a randomised controlled trial, ${ }^{34}$ controlled for treatment and showed it was not a confounder. Of the 12 studies that investigated potential treatment effect modifiers, 11 did not have a control condition or a comparator treatment. It is therefore unclear whether the 22 baseline patient characteristics identified in these studies actually predict success following a specific treatment (treatment effect modifiers) or are just non-specific prognostic factors. As a result of these limitations, pooling and meta-analyses of the data were not warranted. Although definitive conclusions cannot be drawn until these methodological limitations are addressed, in order to make the best use of available evidence, our discussion will focus first on studies investigating prognostic factors for a poor outcome followed by studies that investigated potential treatment effect modifiers.

Prognostic factors are important in the decision-making process and managing patient expectations. ${ }^{53}$ Persistent PFP symptoms could have a negative impact on the physiological and psychological well-being of an individual with PFP. ${ }^{1} 5455$ Of the 12 studies that evaluated prognostic factors for a poor outcome, 16 characteristics were reported, but only 5 were investigated by more than 3 studies. Longer duration of knee pain, ${ }^{27} 3536$ older age, ${ }^{39}$ greater usual pain severity and lower baseline anterior knee pain score ${ }^{27}$ were factors of an unsuccessful outcome (table 5). Three of the five studies that evaluated duration of knee pain, one a large prospective study, reported a consistent finding of longer duration of pain $\left(>4\right.$ months $\left.^{35}\right)$ as an indicator of a poor outcome. Longer duration of symptoms as a poor prognostic indicator seems to be consistent across musculoskeletal conditions, ${ }^{56}$ even in adolescents with knee pain. ${ }^{1}$ It would appear prudent for clinicians to keep duration of symptoms in mind when consulting a patient with PFP. This prognostic factor should also be considered in guidelines and future research for the effective management/prevention of persistent PFP.

Twelve studies evaluated potential treatment effect modifiers associated with a successful outcome after a specific treatment with four conducting multivariate analyses to report clinical prediction rules for either foot orthoses ${ }^{30} 33$ or lumbopelvic manipulation. ${ }^{34} 48$ A limitation of these single group studies identified in the QUADCPR appraisal was (1) the absence of an 


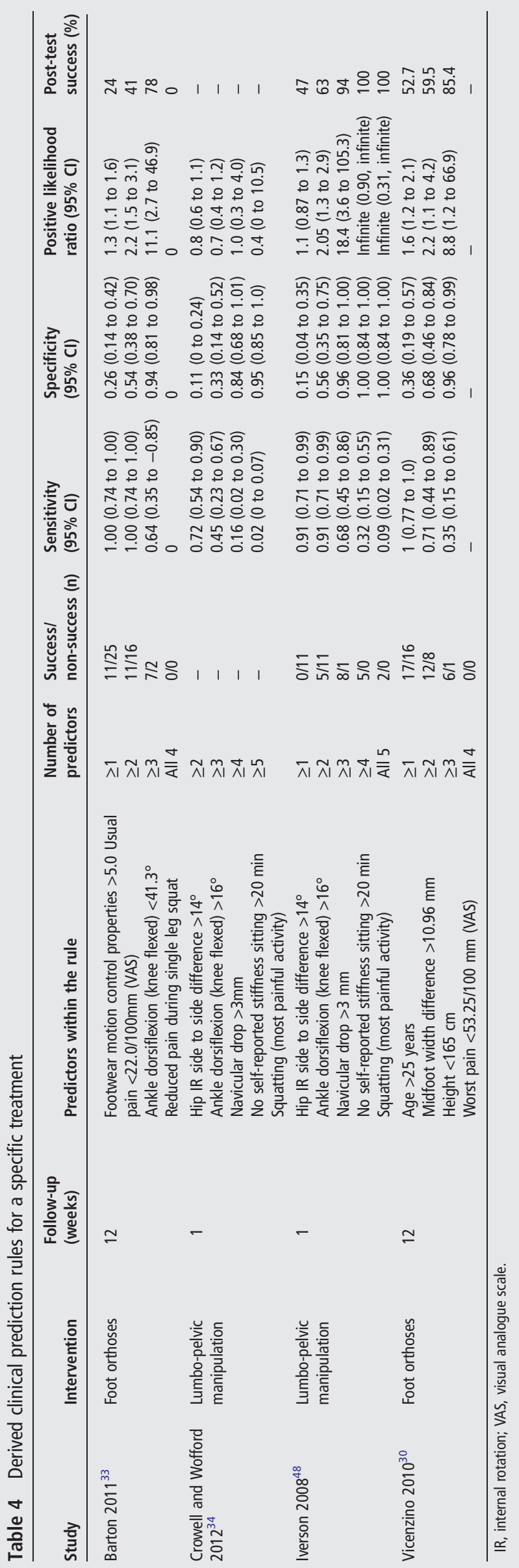


Table 5 Prognostic factors and potential treatment effect modifiers identified in this review

\begin{tabular}{|c|c|c|c|}
\hline & Modifiable with non-operative treatment & Potentially modifiable & $\begin{array}{l}\text { Unable to modify with } \\
\text { non-operative treatment }\end{array}$ \\
\hline \multicolumn{4}{|l|}{ Prognosis } \\
\hline Clinically measurable & $\begin{array}{l}\text { Swelling of knee (self-reported) } \\
\text { Lower Kujala score }^{27} \\
\text { Higher frequency of pain } \\
\text { Bilateral symptoms } \\
\text { Lower eccentric knee strength } \\
\text { Lo } \\
\text { Larger asymmetry in side-to-side isometric } \\
\text { quads strength }\end{array}$ & $\begin{array}{l}\text { Low/middle education }{ }^{37} \\
\text { Poor health }{ }^{37}\end{array}$ & $\begin{array}{l}\text { Longer duration } 273536 \\
\text { Older age } \\
\text { Female gender }^{36} \\
\text { Patellar hypermobility }^{36}\end{array}$ \\
\hline Not standard clinical measurement & Smaller quads cross sectional area $a^{38}$ & Slower VMO reflex response ${ }^{35}$ & $\begin{array}{l}\text { Tibial tubercle lateral } \\
\text { deviation }>14.6 \mathrm{~mm}^{41} \\
\text { Chondromalacia patella }^{41}\end{array}$ \\
\hline \multicolumn{4}{|l|}{ Foot orthoses } \\
\hline Clinically measurable & $\begin{array}{l}\text { Midfoot width difference }>11 \mathrm{~mm}^{30} 32 \\
\text { Ankle dorsiflexion (knee flexed) }<41.3^{\circ 33} \\
\text { Usual pain }<22 / 100 \mathrm{~mm} \text { (VAS) } \\
\text { Worst pain }<53 / 100 \mathrm{~mm} \text { (VAS) } \\
\text { Reduced pain during single leg squat with } \\
\text { orthoses fitted } \\
\text { Footwear motion control properties (weighted } \\
\text { mean) }<5^{33}\end{array}$ & - & $\begin{array}{l}\text { Height }<165 \mathrm{~cm}^{30} \\
\text { Age }>25 \text { years }^{30}\end{array}$ \\
\hline Not standard clinical measurement & $\begin{array}{l}\text { Greater rearfoot eversion relative to floor }{ }^{46} \\
\text { Reduced medial-lateral peak force during } \\
\text { drop-jump }{ }^{47}\end{array}$ & & \\
\hline \multicolumn{4}{|l|}{ Patellar taping } \\
\hline Clinically measurable & Lower body mass index ${ }^{31}$ & & Larger Q-angle ${ }^{31}$ \\
\hline Not standard clinical measurement & & Smaller lateral patellofemoral angle ${ }^{31}$ & \\
\hline \multicolumn{4}{|c|}{ Leg press and stretching lower limb muscles } \\
\hline Not standard clinical measurement & & $\begin{array}{l}\text { Difference in patellar tilt angle between } \\
\text { maximum quadriceps contraction and } \\
\text { quadriceps relaxed }^{28}\end{array}$ & - \\
\hline
\end{tabular}

appropriate comparator intervention, ${ }^{57}$ and (2) inadequate statistical power because limiting sample sizes were too small. Of the 12 studies that investigated potential treatment effect modifiers, 11 did not have a control condition or a comparator treatment. The lack of a control condition or a comparator treatment means there is no way to know that the outcome was necessarily due to the specific treatment. It is therefore unclear whether the baseline patient characteristics identified in these studies actually predict success following a specific treatment (treatment effect modifiers) or are just non-specific prognostic factors. The risk of spurious findings when overfitting or underfitting data to the limiting sample size was highlighted in a replication study of lumbopelvic manipulation for $\mathrm{PFP}^{34}$ which used the same methods as the original study ${ }^{48}$ but achieved contrasting results. Replication studies play an important role in predictive performance in a second, independent sample, especially when there are concerns about overfitting/underfitting data in the original study because of a relatively small limiting sample size. These studies need careful consideration in design that allow for an analysis of the interaction between treatment group and status on the prediction rule.

Foot orthoses were the most common treatment in studies that attempted to identify potential treatment effect modifiers (six studies). One factor that was identified by two studies ${ }^{30} 32$ and formed part of a multivariate clinical prediction rule was midfoot width difference of $>11 \mathrm{~mm}$, reported by Mills et al. ${ }^{32}$ This study was the only 1 of the 13 studies looking at treatment effect modifiers to use a control group (wait-and-see approach). ${ }^{32}$ Mills et $a l^{32}$ provide preliminary evidence that midfoot width difference might be useful at identifying those who might benefit from a foot orthosis, beyond natural history of the condition in the short term. This provides the clinician some useful information/evidence beyond any prognostic value of this foot characteristic.

While single-group studies cannot distinguish between treatment effect modifiers and non-specific prognostic factors, they can identify prognostic factors that could potentially be treatment effect modifiers. Rather appropriately the bulk of identified factors that might predict success with foot orthoses were based at or around the foot. The two studies reporting clinical prediction rules for prescribing foot orthoses reported likelihood ratios that could signify a moderate to large and often conclusive shift in probability of a successful treatment. ${ }^{26}$ The factors that are most likely to be clinically modifiable are ankle dorsiflexion (tibial inclination $<41.3^{\circ}$ from the vertical), ${ }^{33}$ midfoot width difference $(>11 \mathrm{~mm})^{30} 32$ and footwear motion control properties (weighted mean $>5$ ). ${ }^{33}$ An interesting clinical examination finding that contributed to one of these clinical prediction rules was a positive treatment direction test, ${ }^{58}$ which is essentially the immediate reduction in pain with a single leg squat on initial wearing of a foot orthosis. ${ }^{33}$ Somewhat consistent with the report of a positive treatment direction test ${ }^{33}$ is the finding of an immediate decrease in medial-to-lateral peak foot force with fitting a foot orthosis being associated with a successful outcome. ${ }^{47}$ In another laboratory study, kinematic analysis found those with greater rearfoot eversion relative to the floor would also successfully respond to foot orthoses. ${ }^{46}$ Taking the findings collectively, there appears to be a body of exploratory results from single-group studies that suggests the ability of the reported clinically measureable and modifiable prognostic 
factors to be potential treatment effect modifiers to identify those who would be successful with foot orthoses (table 5). Further research is necessary to investigate clinically relevant and plausible prognostic factors from single group studies in appropriately designed clinical trials to test for their ability to be treatment effect modifiers. In particular, midfoot width difference should be further explored as a treatment effect modifier for foot orthoses compared with other treatments because it has been shown to predict success with foot orthoses when compared with no intervention.

In clinical practice, a positive finding of reproduction of symptoms with a femoral slump test, that reduced with neck extension, would reasonably direct the clinician to consider using femoral nerve mobilisations in patients who have PFP. Only one study reported clinical features that predicted success after femoral nerve mobilisation treatment. ${ }^{49}$ In addition to the limitations of the single-group design, the authors used a modified testing protocol for the femoral slump test that is not easy to replicate in a timely manner in clinical practice. It is questionable the degree of confidence with which a clinician could determine a $3^{\circ}$ difference from side to side as the authors did not report the error of this test measurement, so it is difficult to know if $3^{\circ}$ exceeds measurement error. Nevertheless, further investigation of this treatment approach is warranted.

Patellar taping, strengthening and stretching exercises of the thigh and lower limb muscles are often recommended to treat PFP. $^{52}$ A study by Lan et $a l^{31}$ reported that success following patellar taping was associated with lateral patellofemoral angle and Q angle. Peng and Song ${ }^{28}$ measured the difference in patellar tilt angle between relaxed and contracted states of the quadriceps, noting a greater difference, that is, greater realignment of the patella with contracted quads with treatment success. Notably though, all these measures from single studies of patellar position were made with radiological imaging, which is not readily accessible in a typical clinical setting (table 5).

A series of important methodological issues were identified in the reviewed studies. Prognostic studies should ideally be prospective in design, ${ }^{12}$ but in order to review all available studies that reported prognostic factors, weaker-designed retrospective studies were also included in this review. Studies investigating predictors of a successful outcome after a specific treatment usually analysed too many potential predictors for the limiting sample size. This increases the risk of overfitting (or underfitting) the data which can lead to the identification of predictors that are implausible and likely to perform poorly in new samples of patients. ${ }^{29}$ The absence of comparator interventions in studies investigating outcomes after a specific treatment makes it difficult to differentiate between treatment effect modifiers and non-specific prognostic factors. Future studies should determine a sufficient limiting sample size to order to guide recruitment of an appropriate sample size. ${ }^{25}$ Finally, studies need to apply appropriate blinding where possible for participants and treating clinicians, but it is critical to blind investigators assessing the outcome to minimise false positives, or negatives and potential biases.

\section{CONCLUSION}

This review of relationships between patient characteristics and treatment outcomes for PFP identified that methodological limitations such as the absence of a control/comparator group, or too many predictors for the limiting sample size, make it unclear whether the predictors reported actually modify treatment effects. Despite the limitations inherent in current research evidence, we identified modifiable and measurable factors that have been studied so as inform hypotheses that may help in the clinical decision-making process (table 5). Three prognostic studies of patient characteristics identified that persistence of PFP beyond 4 months should alert clinicians to increased risk of a poor outcome. Greater change in midfoot width from non-weight-bearing to weight-bearing was the only characteristic that had sufficient evidence for being a potential treatment effect modifier for a successful outcome after foot orthoses treatment. The LR+ suggested a small but sometimes important shift in probability of a successful outcome, and this characteristic did not predict short-term improvement in a control group who received no intervention. Adequately powered randomised trials that compare relevant treatments are needed, so that treatment can be tailored to the individual patient.

\section{What are the findings?}

- Longer duration of patellofemoral pain symptoms ( $>4$ months) was the most reported prognostic factor of a poor outcome.

- Potential treatment effect modifiers were identified in this review that warrant further investigation.

- Preliminary evidence suggests greater midfoot width mobility may predict a better outcome to foot orthoses treatment.

\section{How might it impact on clinical practice in the near}

\section{future?}

- Duration of symptoms should be considered in making informed clinical decisions regarding outcome expectations.

- Methodological deficiencies of studies limit clinical decisions of predicted outcomes between treatment options.

- More mobile feet might do better with foot orthoses as a management strategy.

- Studies addressing methodological deficiencies will provide better informed decisions regarding predicted outcomes and treatment effects.

Twitter Follow Mark Matthews @Mark_MatthewsNZ, @MichealRathleff, @kaycrossley, @Bill_Vicenzino

Contributors MM, MSR, AC, KCP, RN, TM and BV conceived and designed the review. MM executed the search. MM, MSR and BV screened the articles, extracted the data and rated the methodological quality of the studies. MM, MSR, AC and BV performed the analysis and drafted the manuscript. MM, MSR, AC, KCP, RN, TM and $\mathrm{BV}$ interpreted the data, revised the manuscript and approved the final version.

Funding NHMRC programme grant (number 1091302) funded the review. MM is funded by the Australian Postgraduate Award.

Competing interests None declared.

Provenance and peer review Not commissioned; externally peer reviewed.

\section{REFERENCES}

1 Rathleff MS, Rathleff CR, Olesen JL, et al. Is knee pain during adolescence a self-limiting condition? Prognosis of patellofemoral pain and other types of knee pain. Am J Sports Med 2016;44:1165-71.

2 Collins NJ, Bierma-Zeinstra SM, Crossley KM, et al. Prognostic factors for patellofemoral pain: a multicentre observational analysis. $\mathrm{Br}$ J Sports Med 2013;47:227-33.

3 Mølgaard C, Rathleff MS, Simonsen O. Patellofemoral pain syndrome and its association with hip, ankle, and foot function in 16- to 18-year-old high school 
students: a single-blind case-control study. J Am Podiatr Med Assoc 2011;101:215-22.

4 Rathleff MS, Roos EM, Olesen JL, et al. High prevalence of daily and multi-site pain - a cross-sectional population-based study among 3000 Danish adolescents. BMC Pediatr 2013;13:191.

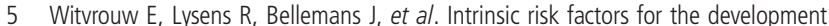
of anterior knee pain in an athletic population: a two-year prospective study. Am J Sports Med 2000:28:480-9.

6 Boling M, Padua D, Marshall S, et al. Gender differences in the incidence and prevalence of patellofemoral pain syndrome. Scand J Med Sci Sports 2010:20:725-30.

7 Roush JR, Curtis Bay R. Prevalence of anterior knee pain in 18-35 year-old females. Int J Sports Phys Ther 2012;7:396-401.

8 Nimon G, Murray D, Sandow M, et al. Natural history of anterior knee pain: a 14- to 20-year follow-up of nonoperative management. J Pediatr Orthop 1998;18:118-22.

9 Lack S, Barton C, Vicenzino B, et al. Outcome predictors for conservative patellofemoral pain management: a systematic review and meta-analysis. Sports Med 2014;44:1703-16.

10 Lankhorst NE, Bierma-Zeinstra SM, van Middelkoop M. Factors associated with patellofemoral pain syndrome: a systematic review. Br J Sports Med 2013;47:193-206.

11 Barton CJ, Lack S, Hemmings $S$, et al. The best practice guide to conservative management of patellofemoral pain: incorporating level 1 evidence with expert clinical reasoning. Br J Sports Med 2015:49:923-34.

12 Moons KGM, Royston $P$, Vergouwe $Y$, et al. Prognosis and prognostic research: what, why, and how? BMJ 2009:338:b375.

13 Collins GS, Reitsma JB, Altman DG, et al. Transparent reporting of a multivariable prediction model for individual prognosis or diagnosis (TRIPOD): the TRIPOD Statement. BMC Med 2015;13:b375.

14 Hancock M, Herbert RD, Maher CG. A guide to interpretation of studies investigating subgroups of responders to physical therapy interventions. Phys The 2009;89:698-704.

15 Moher D, Liberati A, Tetzlaff J. Preferred reporting items for systematic reviews and meta-analyses: the PRISMA statement. Ann Intern Med 2009:151:264-9.

16 Heintjes EM, Berger M, Bierma-Zeinstra SM, et al. WITHDRAWN: Exercise therapy for patellofemoral pain syndrome. Cochrane Database Syst Rev 2015(1):CD003472.

17 van der Heijden RA, Lankhorst NE, van Linschoten R, et al. Exercise for treating patellofemoral pain syndrome. Cochrane Database Syst Rev 2015;1:CD010387.

18 Genaidy AM, LeMasters GK. The epidemiological appraisal instrument (EAI): a brief overview. Theor Issues Ergon Sc 2006;7:187-9.

19 Nix SE, Vicenzino BT, Collins NJ, et al. Characteristics of foot structure and footwear associated with hallux valgus: a systematic review. Osteoarthr Cartil 2012:20:1059-74.

20 Rathleff MS, Rathleff CR, Crossley KM, et al. Is hip strength a risk factor for patellofemoral pain? A systematic review and meta-analysis. Br J Sports Med 2014;48:1088

21 Genaidy AM, Lemasters GK, Lockey J, et al. An epidemiological appraisal instrument-a tool for evaluation of epidemiological studies. Ergonomics 2007:50:920-60.

22 Cook C, Brismée JM, Pietrobon R, et al. Development of a quality checklist using Delphi methods for prescriptive clinical prediction rules: the QUADCPR. J Manipulative Physiol Ther 2010;33:29-41.

23 Moons KG, Altman DG, Reitsma JB, et al. Transparent Reporting of a multivariable prediction model for Individual Prognosis or Diagnosis (TRIPOD): explanation and elaboration. Ann Intern Med 2015;162:W1-73.

24 Cook C, Hegedus E, Hawkins R, et al. Diagnostic accuracy and association to disability of clinical test findings associated with patellofemoral pain syndrome. Physiother Can 2010:62:17-24.

25 Hancock MJ, Kjaer P, Korsholm L, et al. Interpretation of subgroup effects in published trials. Phys Ther 2013;93:852-9.

26 Jaeschke R, Guyatt GH, Sackett DL, et al. Guides to the medical literature: III. How to use an article about a diagnostic test $B$. What are the results and will they help me in caring for my patients? JAMA 1994;271:703-7.

27 Collins NJ, Crossley KM, Darnell R, et al. Predictors of short and long term outcome in patellofemoral pain syndrome: a prospective longitudinal study. BMC Musculoskelet Disord 2010;11:11.

28 Peng $\mathrm{HT}$, Song $\mathrm{CY}$. Predictors of treatment response to strengthening and stretching exercises for patellofemoral pain: an examination of patellar alignment. Knee 2015:22:494-8.

29 Vittinghoff $\mathrm{E}, \mathrm{McCulloch} \mathrm{CE}$. Relaxing the rule of ten events per variable in logistic and Cox regression. Am J Epidemiol 2007:165:710-8.

30 Vicenzino B, Collins NJ, Cleland J, et al. A clinical prediction rule for identifying patients with patellofemoral pain who are likely to benefit from foot orthoses: a preliminary determination. Br J Sports Med 2010;44:862-6.
31 Lan TY, Lin WP, Jiang CC, et al. Immediate effect and predictors of effectiveness of taping for patellofemoral pain syndrome: a prospective cohort study. Am J Sports Med 2010;38:1626-30.

32 Mills $\mathrm{K}$, Blanch $\mathrm{P}$, Dev $\mathrm{P}$, et al. A randomised control trial of short term efficacy of in-shoe foot orthoses compared with a wait and see policy for anterior knee pain and the role of foot mobility. Br J Sports Med 2012:46:247-52.

33 Barton CJ, Menz HB, Crossley KM. Clinical predictors of foot orthoses efficacy in individuals with patellofemoral pain. Med Sci Sports Exerc 2011:43:1603-10.

34 Crowell MS, Wofford NH. Lumbopelvic manipulation in patients with patellofemoral pain syndrome. J Man Manip Ther 2012;20:113-20.

35 Witvrouw E, Lysens R, Bellemans J, et al. Which factors predict outcome in the treatment program of anterior knee pain? Scand J Med Sci Sports 2002:12:40-6.

36 Blønd L, Hansen L. Patellofemoral pain syndrome in athletes: a 5.7-year retrospective follow-up study of 250 athletes. Acta Orthop Belg 1998;64:393-400.

37 Kastelein M, Luijsterburg PAJ, Heintjes EM, et al. The 6-year trajectory of non-traumatic knee symptoms (including patellofemoral pain) in adolescents and young adults in general practice: a study of clinical predictors. Br J Sports Med 2015;49:400-5

38 Pattyn E, Mahieu N, Selfe J, et al. What predicts functional outcome after treatment for patellofemoral pain? Med Sci Sports Exerc 2012;44:1827-33.

39 Kannus P, Niittymaki S. Which factors predict outcome in the nonoperative treatment of patellofemoral pain syndrome? A prospective follow-up study. Med Sci Sports Exerc 1994;26:289-96.

40 Natri $A$, Kannus $P$, Jarvinen $M$. Which factors predict the long-term outcome in chronic patellofemoral pain syndrome? A 7-yr prospective follow-up study. Med Sci Sports Exerc 1998;30:1572-7.

41 Wittstein JR, O'Brien SD, Vinson EN, et al. MRI evaluation of anterior knee pain: predicting response to nonoperative treatment. Skeletal Radiol 2009;38: 895-901.

42 Karlsson J, Thomeé R, Swärd L. Eleven year follow-up of patello-femoral pain syndrome. Clin J Sport Med 1996;6:22-6.

43 Kettunen JA, Harilainen A, Sandelin J, et al. Knee arthroscopy and exercise versus exercise only for chronic patellofemoral pain syndrome: 5-year follow-up. $\mathrm{Br} J$ Sports Med 2012;46:243-6.

44 Rathleff MS, Roos EM, Olesen JL, et al. Self-reported recovery is associated with improvement in localised hyperalgesia among adolescent females with patellofemoral pain - results from a cluster randomised trial. Clin J Pain 2015;32:428-34

45 Sutlive TG, Mitchell SD, Maxfield SN, et al. Identification of individuals with patellofemoral pain whose symptoms improved after a combined program of foot orthosis use and modified activity: a preliminary investigation. Phys Ther 2004:84:49-61.

46 Barton CJ, Menz HB, Levinger $\mathrm{P}$, et al. Greater peak rearfoot eversion predicts foot orthoses efficacy in individuals with patellofemoral pain syndrome. Br J Sports Med 2011:45:697-701

47 Rathleff MS, Richter C, Brushøj C, et al. Custom-made foot orthoses decrease medial foot loading during drop jump in individuals with patellofemoral pain. Clin J Sport Med 2015:26:335-7.

48 Iverson CA, Sutlive TG, Crowell MS, et al. Lumbopelvic manipulation for the treatment of patients with patellofemoral pain syndrome: development of a clinica prediction rule. J Orthop Sports Phys Ther 2008;38:297-312.

49 Huang BY, Shih YF, Chen WY, et al. Predictors for identifying patients with patellofemoral pain syndrome responding to femoral nerve mobilization. Arch Phys Med Rehabil 2015:96:920-7.

50 Lankhorst NE, van Middelkoop M, van Trier YDM, et al. Can we predict which patients with patellofemoral pain are more likely to benefit from exercise therapy? A secondary exploratory analysis of a randomized controlled trial. J Orthop Sports Phys Ther 2015;45:183-9.

51 Barton CJ, Bonanno D, Menz HB. Development and evaluation of a tool for the assessment of footwear characteristics. J Foot Ankle Res 2009;2:10

52 McConnell J. The management of chondromalacia patellae: a long term solution. Aust J Physiother 1986;32:215-23.

53 Crossley KM, Callaghan MJ, van Linschoten R. Patellofemoral pain. BMJ 2015;351: h3939.

54 Vlaeyen JW, Linton SJ. Fear-avoidance and its consequences in chronic musculoskeletal pain: a state of the art. Pain 2000:85:317-32.

55 Finnoff JT, Hall MM, Kyle K, et al. Hip strength and knee pain in high school runners: a prospective study. $P M R$ 2011;3:792-801.

56 Mallen $C D$, Peat $G$, Thomas $E$, et al. Prognostic factors for musculoskeletal pain in primary care: a systematic review. Br J Gen Pract 2007:57:655-61.

57 Kent $\mathrm{P}$, Hancock M, Petersen DHD, et al. Clinimetrics corner: choosing appropriate study designs for particular questions about treatment subgroups. J Man Manip Ther 2010:18:147-52.

58 Vicenzino B. Foot orthotics in the treatment of lower limb conditions: a musculoskeletal physiotherapy perspective. Man Ther 2004:9:185-96. 